\title{
Benchmarking the prognostic performance of EUROSCORE II with the original EuroSCORE in cardiac surgery patients
}

Tsaousi G., Pitsis A., Pourzitaki Ch., Lolakos K., Vasilakos D.

Department of Anesthesiology, Aristotle University of Thessaloniki, Faculty of Medicine, Thessaloniki, Greece

\section{Background and Goal of Study}

The European System for Cardiac Operative Risk Evaluation (EuroSCORE) is one of the most documented preoperative risk algorithms for assessing operative morbidity and mortality following cardiac surgery, which has been recently refined to EuroSCORE II, in order to improve the performance of the original EuroSCORE. Aim of this study was to comparably assess the applicability of the updated EuroSCORE II model with the logistic (log) and additive (add) versions of the initial EuroSCORE, in cardiac surgical population, on the basis of morbidity and mortality.

\section{Materials and Methods}

Prospective, observational study enrolling a total of 1058 consecutive patients (aged 63.9 \pm 9.1 years, male to female ratio: 3.7/1), admitted to our post-cardiac surgery intensive care unit (ICU) following cardiosurgical procedure, were included in this data analysis study. EuroSCORE add, EuroSCORE log and EuroSCORE II were calculated for each participant. The study endpoints were operative mortality (30-day) and morbidity assessed by length of intensive care unit stay (ICU-LOS), using 2days as cut-off point. For statistical purposes Mann-Whitney test and ROC curve analysis were used as appropriate.

\section{Results}

Overall ICU mortality of the study population was $3.3 \%(n=35)$. ICU-LOS equal or less than 2 days was recorded in $74.1 \%$ ( $\mathrm{n:784})$ participants. Univariate analysis revealed a significant augmentation $(\mathrm{p}=0.000)$ of the studied parameters in patients with ICU-LOS > 2 days and in those with poor outcome (Fig.1 \& 2). In terms of ICU-LOS, ROC curve analysis showed that EuroSCORE II had the best performance (AUC 0.720; cut-off 1.76), followed by EuroSCORE log (AUC 0.695; cut-off 4.45) and EuroSCORE add (AUC 0.687; cut-off 5). In terms of operative mortality, the prognostic ability of these algorithms was further improved for EuroSCORE II (AUC 0.843; cut-off 3.44), for EuroSCORE log (AUC 0.827; cut-off 3.67) and for EuroSCORE add (AUC 0.818; cut-off 7). Data from ROC curve analysis are presented in Figure 3.

cur-olr (), vala Irom ruv curve analysis are presented in rigure 3.

Figure 3. ROC curve analysis in terms of ICU-LOS and mortality.
ICU LOS

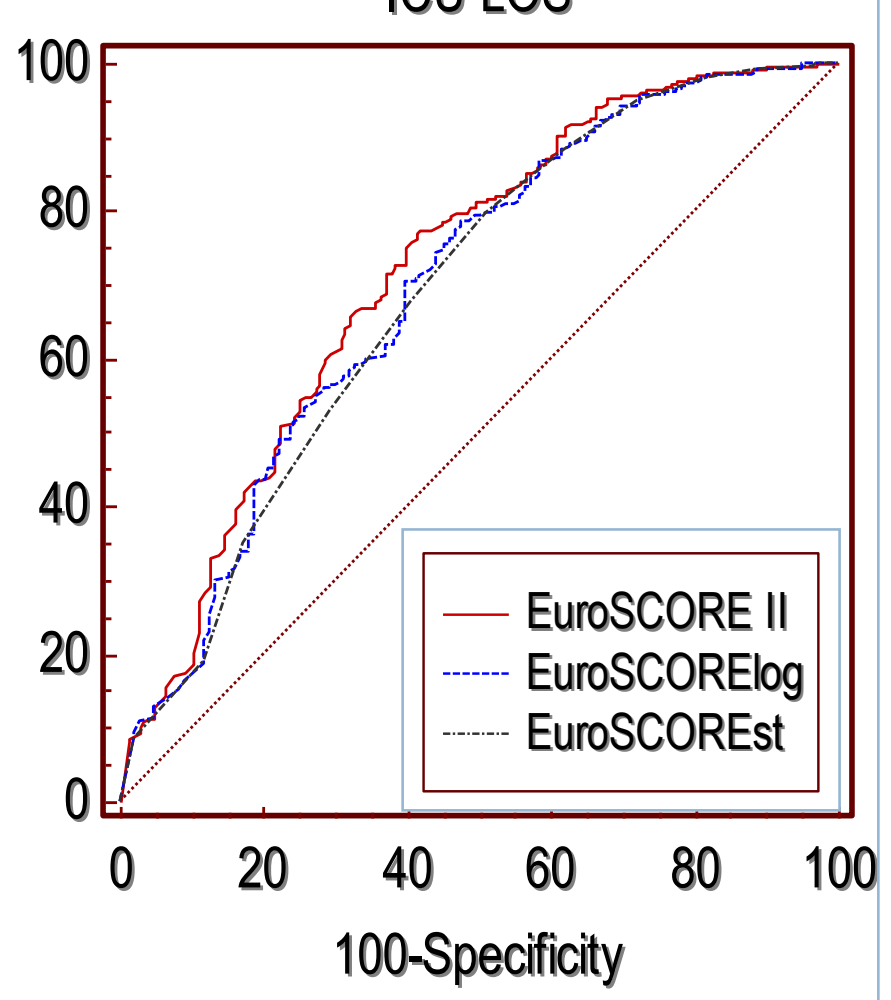

MORTALITY

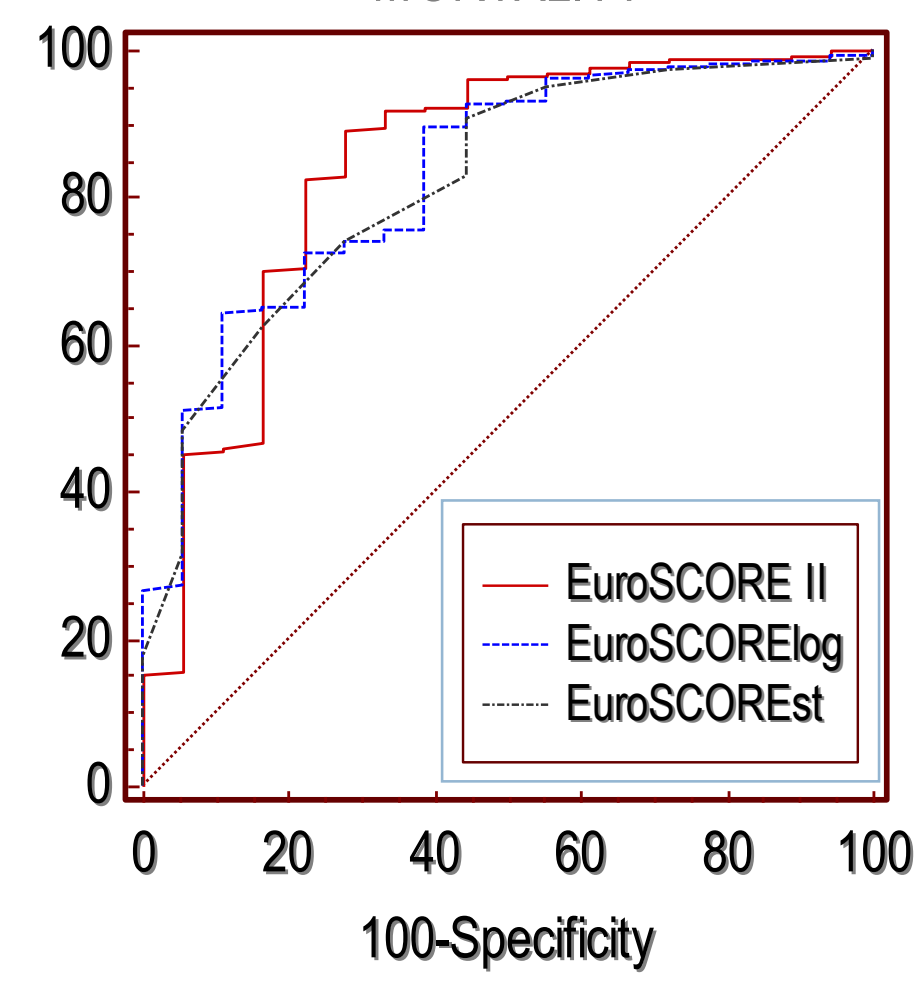

Refierences

1. Nashef SA, et al. EuroSCORE II. Eur J Cardiothorac Surg, 2012 ;41(4):734-44.
Figure 1. Bar-chart of Euroscore derivates according to length of ICU stay. Data are presented as mean.

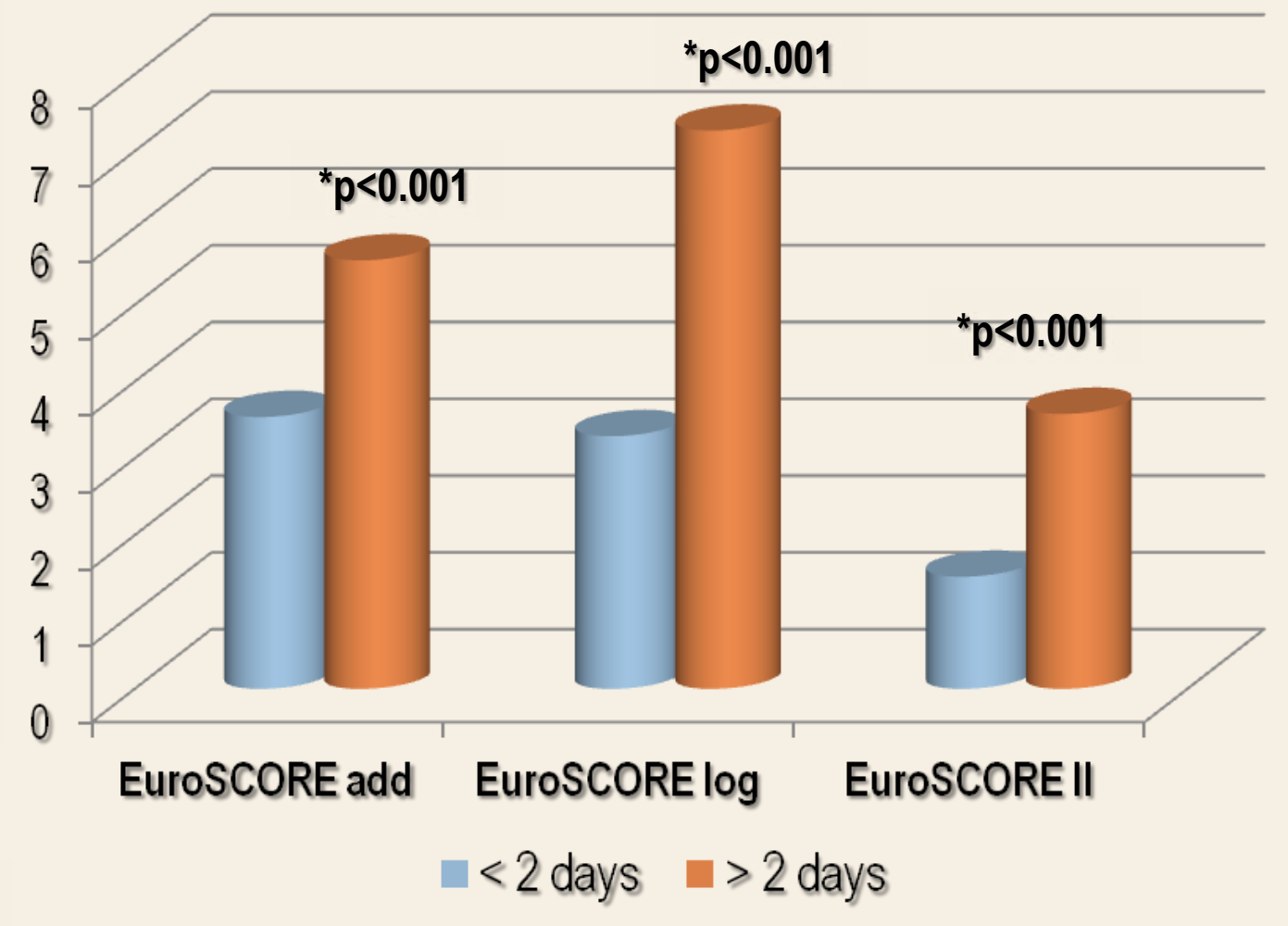

Figure 2. Bar-chart of Euroscore derivates according to length of ICU stay. Data are presented as mean.

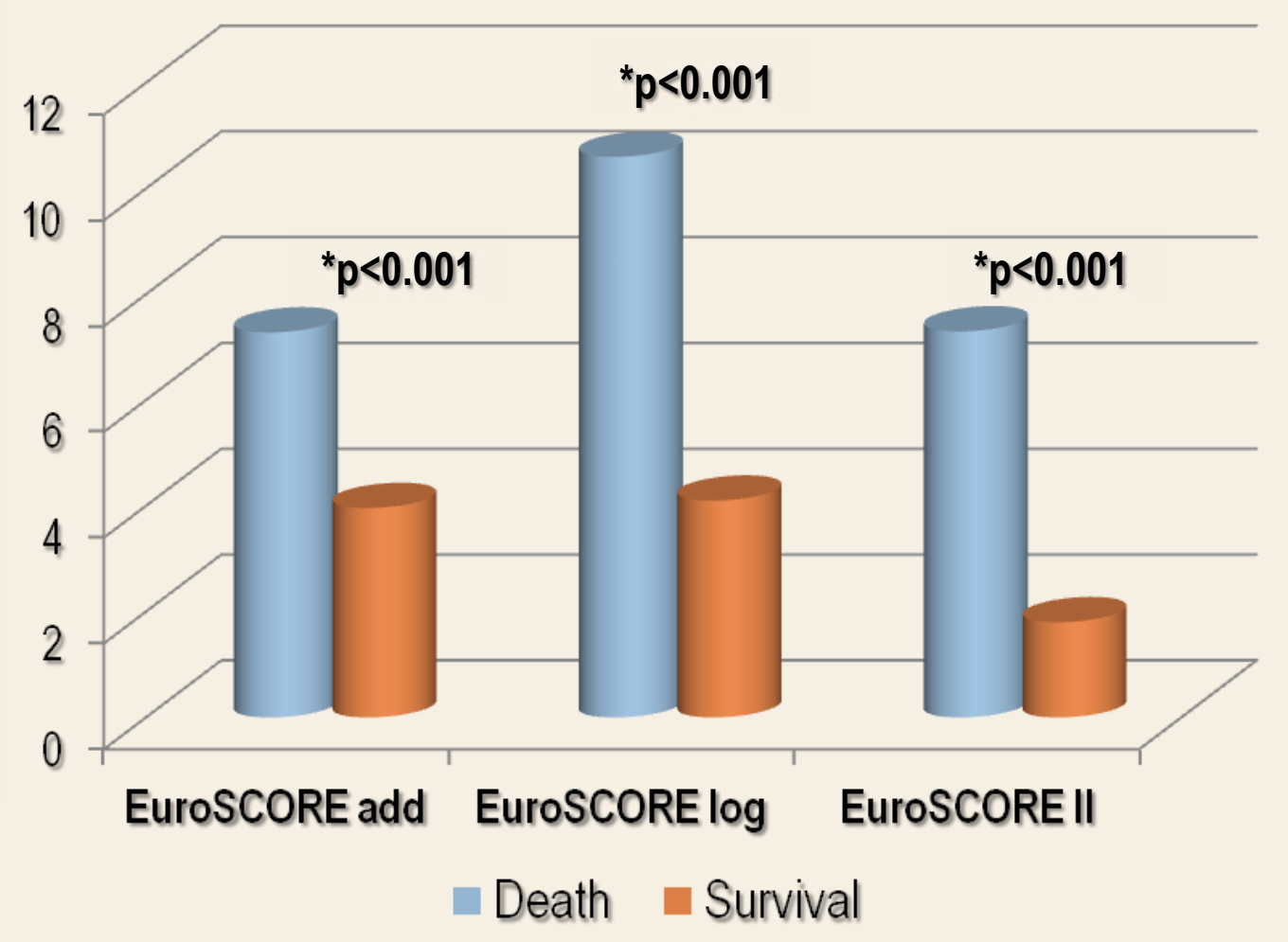

Conclusion

The findings of the present study substantiate that EuroScore log, EuroSCORE add and EuroSCORE II models, confer noteworthy prognostic value when applied to adult cardiac surgical population, being more pronounced in terms of mortality. The updated EuroSCORE II risk model seems to confer superior discriminatory performance compared to the original EuroSCORE. Thus, the implementation of EuroSCORE II in cardiac ICU could possibly contribute towards risk stratification, mortality prediction and identification of patients who are at increased risk for prolonged ICU stay. 